\title{
Second Workshop on Prediction Markets
}

\author{
Yiling Chen \\ Yahoo! Research \\ New York, NY, USA \\ David M. Pennock \\ Yahoo! Research \\ New York, NY, USA
}

\author{
John O. Ledyard \\ California Institute of Technology \\ Pasadena, CA, USA \\ Eric Zitzewitz \\ Stanford University \\ Stanford, CA, USA
}

\section{Categories and Subject Descriptors}

J.4 [Computer Applications]: Social and Behavioral Sciences-Economics

\section{General Terms}

Economics, Theory, Algorithms

\section{Keywords}

Prediction market, information aggregation

\section{WORKSHOP OUTLINE}

The Second Workshop on Prediction Markets, following the success of the first workshop held in 2005, explores research challenges in using markets as predictive devices. The workshop brings together researchers and practitioners from a variety of relevant fields, including economics, finance, computer science, and statistics, in both academia and industry, to discuss the state of the art today, and the challenges and prospects for tomorrow in the field of prediction markets.

Most financial markets are designed to satisfy demand for trade, and price discovery is a beneficial side effect. In prediction markets, price discovery is the designer's end goal and trading is a means to that end. For example, suppose a policymaker seeks a forecast of the likelihood of an avian flu outbreak in 2008. She may float a security paying $\$ 1$ if and only if an outbreak actually occurs in 2008, hoping to attract traders willing to speculate on the outcome. With sufficient liquidity, traders will converge to a consensus price reflecting their collective information about the value of the security, which in this case directly corresponds to the probability of outbreak. Empirically, prediction markets often yield better forecasts than other methods across a diverse array of settings. Related academic work dates to Hayek in 1945 and has its roots in the efficient market hypothesis in finance. The past decade has seen a heathy growth in the field, including a sharp rise in publications and events, and the creation of the Journal of Prediction Markets. Academic work includes mechanism design, experimental (laboratory) studies, field studies, and empirical analyses. In industry, several companies including Eli Lilly, Corning, HP, Microsoft, and Google have piloted internal

Copyright is held by the author/owner(s).

EC'07, June 11-15, 2007, San Diego, California, USA.

ACM 978-1-59593-653-0/07/0006. prediction markets. Other companies, including ConsensusPoint, InklingMarkets, NewsFutures, and TradeSports, base their business on providing public prediction markets, prediction market software solutions, or consulting services. The growth of the field is reflected and fueled by a wave of popular press articles and books on the topic, most prominently Surowiecki's "The Wisdom of Crowds".

Despite its growth, the area faces challenges regarding how best to design, deploy, analyze, and understand prediction markets. A variety of interesting research topics can help bring the community of researchers and practitioners together. One important research direction is designing mechanisms for prediction markets, especially for events with a combinatorial outcome space. Technical challenges in this direction include the thin market problem and computational issues of order matching, among others. Another notable issue is manipulation in prediction markets. Understanding the effect of manipulation is especially important for prediction markets to find their way to assist individuals and organizations in making critical decisions. Prediction markets face social and political obstacles including antigambling laws and moral and ethical concerns, both real and constructed.

Accepted research contributions and invited contributions from a rich set of experimental, empirical, and theoretical perspectives in the field of prediction markets are presented at the workshop. Topics include: mechanism design; gametheoretic analysis of mechanisms, behaviors, and dynamics; decision markets; combinatorial prediction markets; market makers for prediction markets; manipulation and prediction markets; order matching algorithms; computational issues of prediction markets; liquidity and thin markets; laboratory experiments; empirical analysis; prediction market modeling; industry and field experience; simulations; policy applications and implications; internal corporate applications; and legal and ethical issues. The workshop concludes with panel discussions and open discussions.

\section{PROGRAM COMMITTEE}

- Kay-Yut Chen, HP

- Ely Dahan, University of California, Los Angeles

- Leslie Fine, HP

- Lance Fortnow, University of Chicago

- C. Lee Giles, The Pennsylvania State University 
- Robin Hanson, George Mason University

- Paul J. Healy, Ohio State University and Carnegie Mellon University

- Chris Hibbert, Zocalo Open Source Prediction Market Software

- Anthony M. Kwasnica, The Pennsylvania State University

- Tracy Mullen, The Pennsylvania State University

- Forrest Nelson, University of Iowa
- George Neumann, University of Iowa

- Ryan Oprea, University of California, Santa Cruz

- Daniel Reeves, Yahoo! Research

- Rahul Sami, University of Michigan

- Bernd Skiera, University of Frankfurt

- Martin Spann, University of Passau

- Paul C. Tetlock, University of Texas, Austin

- Justin Wolfers, University of Pennsylvania 